\title{
Blood Group O
}

National Cancer Institute

\section{Source}

National Cancer Institute. Blood Group O. NCI Thesaurus. Code C76249.

A blood group indicating the absence on erythrocytes of both the $A$ and $B$ forms of the $H$ Antigen. 